\title{
Flag Gromov-Witten invariants via crystals
}

\author{
Jennifer Morse $\|^{1}$ and Anne Schilling $\|^{2}$ \\ ${ }^{1}$ Department of Mathematics, Drexel University, Philadelphia, PA 19104, USA \\ ${ }^{2}$ Department of Mathematics, University of California, One Shields Avenue, Davis, CA 95616-8633, USA
}

\begin{abstract}
We apply ideas from crystal theory to affine Schubert calculus and flag Gromov-Witten invariants. By defining operators on certain decompositions of elements in the type- $A$ affine Weyl group, we produce a crystal reflecting the internal structure of Specht modules associated to permutation diagrams. We show how this crystal framework can be applied to study the product of a Schur function with a $k$-Schur function. Consequently, we prove that a subclass of 3-point Gromov-Witten invariants of complete flag varieties for $\mathbb{C}^{n}$ enumerate the highest weight elements under these operators.
\end{abstract}

Résumé. Nous appliquons des idées provenant de la théorie des bases cristallines au calcul de Schubert affine et aux invariants de drapeaux de Gromov-Witten. Nous définissons des opérateurs sur certaines décompositions d'éléments de groupes de Weyl affines en type $A$ afin de construire une base cristalline encodant la structure interne des modules de Specht associés aux diagrammes de permutations. Nous montrons comment la structure de crystal permet d'étudier le produit d'une fonction de Schur avec une $k$-fonction de Schur. En conséquence, nous prouvons que la sous-classe des invariants de 3-points de Gromov-Witten d'une variété complète de drapeaux complets pour $\mathbb{C}^{n}$ énumère les éléments de poids maximaux pour ces opérateurs.

Keywords: flag Gromov-Witten invariants, Littlewood-Richardson coefficients, crystal graphs, Specht modules

\section{Introduction}

Crystal theory [8, 9] and Schubert calculus convene beautifully in type $\mathfrak{g l}_{n}$. The crystal is the graph whose vertices are Young tableaux and whose edges are imposed by coplactic operators first introduced by Lascoux and Schützenberger [21, 22]. The number of connected components gives the multiplicity $c_{\lambda, \mu}^{\nu}$ of the irreducible highest weight module $V_{\nu}$ in $V_{\lambda} \otimes V_{\mu}=\bigoplus_{\nu} c_{\lambda, \mu}^{\nu} V_{\nu}$. Describing multiplicities as highest weights (connected components) is the heart of crystal theory.

From the geometric perspective, the problem is to count certain linear subspaces in projective space. Developments in algebraic geometry and topology convert the problem into the computation of intersection numbers of certain subvarieties in the Grassmannian, which in turn is encoded by the structure constants of Schubert classes $\left\{\sigma_{\lambda}\right\}_{\lambda \subset \text { rect }}$ for the cohomology ring of the Grassmannian $\operatorname{Gr}(a, n)$. Finally,

\footnotetext{
*Partially supported by the NSF grants DMS-1001898, DMS-1301695 and a Simons Fellowship.

$\dagger$ Partially supported by the NSF grants DMS-1001256, OCI-1147247 and a Simons Fellowship. Emails: morsej@math.drexel. edu and anne@math. ucdavis. edu
} 
the computation is made concrete with an isomorphism from $H^{*}(\operatorname{Gr}(a, n))$ to the quotient of a polynomial ring under which Schubert classes correspond to Schur functions. The intersection numbers turn out to be coefficients in the Schur expansion of a product of two Schur functions.

In both cases, the solution can be derived from the discovery, dating back to the early 1990's, that the Frobenius image of $V_{\lambda}$ is a Schur function $s_{\lambda}$. Thus the intersection numbers are $c_{\lambda \mu}^{\nu}$ as well. The Littlewood-Richardson (LR) rule to compute $c_{\lambda \mu}^{\nu}$ was formulated in 1934 [25] by counting certain Yamanouchi tableaux, but the first rigorous proof was not seen until Schützenberger's work [31] in 1977.

The main thrust of our work here is to introduce crystals into a generalization of Schubert calculus centered around the affine Grassmannian $\mathrm{Gr}=G(\mathbb{C}((t))) / G(\mathbb{C}[[t]])$ for $G=S L(n, \mathbb{C})$, where $\mathbb{C}[[t]]$ is the ring of formal power series and $\mathbb{C}((t))=C[[t]]\left[t^{-1}\right]$ is the ring of formal Laurent series. Quillen (unpublished) and Garland and Raghunathan [7] showed that Gr is homotopy-equivalent to the group $\Omega S U(n, \mathbb{C})$ of based loops into $S U(n, \mathbb{C})$. The homology and cohomology of the affine Grassmannian thus acquire an algebra structure; it follows from Bott's work [3] that $H_{*}(\mathrm{Gr})$ and $H^{*}(\mathrm{Gr})$ can be identified with a subring $\Lambda_{(n)}$ and a quotient $\Lambda^{(n)}$ of the ring $\Lambda$ of symmetric functions.

On the one hand, using the algebraic nil-Hecke ring construction, Kostant and Kumar [12] studied Schubert bases of $H^{*}(\mathrm{Gr})$ and Peterson [28] studied Schubert bases of $H_{*}(\mathrm{Gr})$. On the other hand, an explicit distinguished basis for $\Lambda_{(n)}$ came out of a study [17] of Macdonald polynomials. It is comprised of elements called $k$-Schur functions, $\left\{s_{w}^{(k)} \mid w \in \tilde{S}_{n}^{0}\right\}$, indexed by the subset of the affine symmetric group $\tilde{S}_{n}=\left\langle s_{0}, s_{1}, \ldots, s_{n-1}\right\rangle$ consisting of affine Grassmannian elements - representatives of minimal length in cosets of $\tilde{S}_{n} / S_{n}$. Here $k=n-1$. It was shown in [19] that the (affine-LR) coefficients in the products

$$
s_{u}^{(k)} s_{w}^{(k)}=\sum_{v \in \tilde{S}_{n}^{0}} c_{u, w}^{v, k} s_{v}^{(k)}
$$

contain all structure constants for a quantum deformation of the cohomology of the Grassmannian tied to string theory (e.g. [13, 34]). A basis (of dual $k$-Schur functions) for $\Lambda^{(n)}$ was also introduced in [19] and therein it was generalized to a family $\left\{\mathcal{F}_{v w^{-1}}\right\}$ to alternately encode the constants by $\mathcal{F}_{v w^{-1}}=$ $\sum_{u \in \tilde{S}_{n}^{0}} c_{w, u}^{v, k} \mathcal{F}_{u}$. The two approaches converged when Lam proved [15] Shimozono's conjecture that the $k$-Schur basis is a set of representatives for the Schubert classes of $H_{*}(\mathrm{Gr})$. The Schubert structure constants in homology exactly match the affine-LR coefficients reducing their calculation to work in $\Lambda$.

In this article, we produce a crystal framework for affine-LR coefficients. The $k$-Schur functions can be characterized using decreasing factorizations of elements in the type- $A$ affine Weyl group. We introduce a set of operators (see Section 3.2 that act on a subclass of these factorizations with at most $\ell$ factors. The resulting graph is a $U_{q}\left(A_{\ell-1}\right)$-crystal (see Theorem 3.5). At a basic level, we find that the crystals support Specht modules of $S_{n}$ associated to permutation diagrams. The Frobenius images of the modules are stable Schubert polynomials (also known as Stanley symmetric functions). The decomposition into irreducible characters, given by the Edelman-Greene correspondence, intertwines with our crystal operators (see Theorem 4.5).

Lam [14] generalized the Stanley symmetric functions to affine Stanley symmetric functions $F_{\widetilde{w}}$, defined for each $\widetilde{w} \in \tilde{S}_{n}$. We discovered that every affine $\widetilde{w} \in \tilde{S}_{n}$ can instead be identified with a skew shape $\nu / \lambda$ (see Definition 5.2. . It follows that each affine Stanley $F_{\widetilde{w}}$ is in fact a dual $k$-Schur function $\mathcal{F}_{w_{\nu} w_{\lambda}^{-1}}$

We find that highest weight elements in the crystal correspond to certain coefficients in the product of a Schur times a $k$-Schur function. These are affine LR-coefficients (1) since $s_{w_{\mu}}^{(k)}$ reduces simply to the 
Schur function $s_{\mu}$ when indexed by an element $w_{\mu} \in \tilde{S}_{n}^{0}$ that can be generated from a strict subset of $\left\{s_{0}, s_{1}, \ldots, s_{n-1}\right\} . S_{\hat{x}}$ denotes the subgroup of $\tilde{S}_{n}$ generated by $\left\{s_{0}, \ldots, s_{n-1}\right\} \backslash\left\{s_{x}\right\}$. More generally, the crystal applies to the translation of such $w_{\mu}$ by any product of $k$-rectangles $R=\prod_{i} R_{i}$.

Theorem 5.6 Let $v, w \in \tilde{S}_{n}^{0}$ and $\mu \subset\left(r^{n-r}\right)$ with $1 \leq r<n$ such that $v w^{-1} \in S_{\hat{x}}$ or $\ell(\mu)=2$. If $\ell(v)-\ell(w) \neq|\mu|$, then $c_{R w_{\mu}, w}^{R v, k}=0$. Otherwise $c_{R w_{\mu}, w}^{R v, k}=\#$ of highest weight factorizations of $v w^{-1}$ of weight $\mu$.

The crystal also connects to several families of intensely studied constants that arise as a subset of affine LR-coefficients such as 3-point Gromov-Witten invariants for complete flags. The quantum cohomology ring was defined not just for Grassmannians, but for any Kähler algebraic manifold $X$. When $X=\mathrm{Fl}_{n}$, as a linear space, $\mathrm{QH}^{*}\left(\mathrm{Fl}_{n}\right)=H^{*}\left(\mathrm{Fl}_{n}\right) \otimes \mathbb{Z}\left[q_{1}, \ldots, q_{n-1}\right]$ for parameters $q_{1}, \ldots, q_{n-1}$. However, the multiplicative structure is defined by $\mathfrak{S}_{u}^{q} *_{q} \mathfrak{S}_{w}^{q}=\sum_{v} \sum_{q}\langle u, w, v\rangle_{d} q^{d} \mathfrak{S}_{w_{0} v}^{q}$, where the structure constants are the 3-point Gromov-Witten invariants of genus 0, constants which count equivalence classes of certain rational curves in $\mathrm{Fl}_{n}$. Peterson asserted that $\mathrm{QH}^{*}(G / P)$ of a flag variety is a quotient of the homology $H_{*}\left(\mathrm{Gr}_{G}\right)$ of the affine Grassmannian up to localization (details carried out in [16]). Consequently, $\langle u, w, v\rangle_{d}$ arise as coefficients in (1) and in particular, when $d=0$, these include the Schubert structure constants (see Theorem 5.7). Our approach also applies to the structure constants of Verlinde (fusion) algebras and positroid varieties. Details as well as an account on related work and proofs of all results are given in the long version of this paper.

Basic notation for affine permutations and crystals is set in Section 2 . In Section 3 we introduce crystal operators on affine factorizations for certain $\widetilde{w} \in \tilde{S}_{n}$. Section 4 shows that $B(\widetilde{w})$ supports the generalized Specht module of a permutation diagram associated to $\widetilde{w}$. In Section 5 , we use the crystal to describe various $k$-Schur structure constants in the product of a Schur function and a $k$-Schur function.

\section{Acknowledgments}

We would like to thank the ICERM program "Automorphic Forms, Combinatorial Representation Theory and Multiple Dirichlet Series" during the spring 2013 and IHES in Orsay for their hospitality, where part of this work was done. Both authors would like to thank the Simons Foundation for sabbatical support. This work benefitted from computations with S AGE [29, 30].

\section{Notation and background}

\subsection{Type A affine Weyl group}

An affine permutation is a bijection $w$ from $\mathbb{Z} \rightarrow \mathbb{Z}$ where $w(i+n)=w(i)+n$ for all $i$. Since $w$ is determined by the tuple of values $[w(1), w(2), \ldots, w(n)]$, called its window, we often use this notation for affine permutations.

Our focus is on the subset $\tilde{S}_{n, r}$ of affine permutations where $\sum_{i=1}^{n}(w(i)-i)=r n$. The special case when $r=0$ (denoted by $\tilde{S}_{n}=\tilde{S}_{n, 0}$ ) gives the affine Weyl group $\tilde{A}_{n-1}$. $\tilde{S}_{n}$ is a Coxeter group generated by $\left\langle s_{0}, s_{1}, \ldots, s_{n-1}\right\rangle$ satisfying the relations $s_{i}^{2}=1, s_{i} s_{i+1} s_{i}=s_{i+1} s_{i} s_{i+1}$ for all $i$, and $s_{i} s_{j}=s_{j} s_{i}$ if $|i-j|>1$, where indices are taken modulo $n$ (we will work $\bmod n$ without further comment).

A word $i_{1} i_{2} \cdots i_{m}$ in the alphabet $[n]=\{0,1, \ldots, n-1\}$ corresponds to the permutation $w=$ $s_{i_{1}} \ldots s_{i_{m}} \in \tilde{S}_{n}$. The length $\ell(w)$ of $\underset{\tilde{S}}{w}$ is defined by the length of its shortest word. Any word of this length is said to be reduced. The set $\tilde{S}_{n}^{0}$ of affine Grassmannian elements is the set of minimal length coset representatives of $\tilde{S}_{n} / S_{n}$, where $S_{n}$ is the finite symmetric group generated by $\left\langle s_{1}, \ldots, s_{n-1}\right\rangle$. In 
fact, $w \in \tilde{S}_{n}^{0}$ if and only if every reduced word for $w$ ends in 0 . Equivalently, an element $w$ is affine Grassmannian when it has an increasing window $w(1)<w(2)<\cdots<w(n)$.

\subsection{Kashiwara crystals}

Kashiwara [8, 10] introduced a crystal as an edge-colored directed graph satisfying a simple set of axioms. Let $\mathfrak{g}$ be a symmetrizable Kac-Moody algebra with associated root, coroot and weight lattices $Q, Q^{\vee}, P$. Let $I$ be the index set of the Dynkin diagram and denote the simple roots, simple coroots and fundamental weights by $\alpha_{i}, \alpha_{i}^{\vee}$ and $\Lambda_{i}(i \in I)$, respectively. There is a natural pairing $\langle\cdot, \cdot\rangle: Q^{\vee} \otimes P \rightarrow \mathbb{Z}$ defined by $\left\langle\alpha_{i}^{\vee}, \Lambda_{j}\right\rangle=\delta_{i j}$.

Definition 2.1 (Axiomatic) A P-weighted I-crystal is a nonempty set $B$ together with maps wt: $B \rightarrow P$ and $\tilde{e}_{i}, \tilde{f}_{i}: B \rightarrow B \cup\{\mathbf{0}\}$ for all $i \in I$ satisfying

1. $\tilde{f}_{i}(b)=b^{\prime}$ is equivalent to $\tilde{e}_{i}\left(b^{\prime}\right)=b$ for $b, b^{\prime} \in B, i \in I$.

2. For $i \in I$ and $b \in B, \operatorname{wt}\left(\tilde{e}_{i} b\right)=\operatorname{wt}(b)+\alpha_{i}$ if $\tilde{e}_{i} b \in B$ and $\operatorname{wt}\left(\tilde{f}_{i} b\right)=\operatorname{wt}(b)-\alpha_{i}$ if $\tilde{f}_{i} b \in B$.

3. For all $i \in I$ and $b \in B$, we have $\varphi_{i}(b)=\varepsilon_{i}(b)+\left\langle\alpha_{i}^{\vee}\right.$, wt $\left.(b)\right\rangle$, where $\varepsilon_{i}(b)=\max \{d \geq 0 \mid$ $\left.\tilde{e}_{i}^{d}(b) \neq \mathbf{0}\right\}$ and $\varphi_{i}(b)=\max \left\{d \geq 0 \mid \tilde{f}_{i}^{d}(b) \neq \mathbf{0}\right\}$.

An axiomatic crystal satisfying Stembridge's axioms [33] corresponds to a $U_{q}(\mathfrak{g})$-representation. We call such a crystal a $U_{q}(\mathfrak{g})$-crystal.

Theorem 2.2 27 24 Let $B$ be a $U_{q}(\mathfrak{g})$-crystal in the category of integrable highest-weight crystals. Then the connected components of $B$ correspond to the irreducible components. In addition, the irreducible components are in bijection with the highest weight vectors.

\section{Crystal operators on affine factorizations}

\subsection{Affine factorizations}

Elements of $\tilde{S}_{n}$ with a decreasing feature play an important role in the geometry of the affine Grassmannian Gr. Let $i_{1} \cdots i_{\ell}$ be a sequence with each $i_{r} \in[n]$. The word $i_{1} \cdots i_{\ell}$ is cyclically decreasing if no number is repeated and $j-1 j$ does not occur as a subword for any $j \in[n]$ (all indices are taken $\bmod n$ ). If $i_{1} \cdots i_{\ell}$ is cyclically decreasing, then we say the permutation $w=s_{i_{1}} \cdots s_{i_{\ell}}$ is cyclically decreasing.

The focal point of our study is a set of distinguished products of cyclically decreasing elements. For any composition $\alpha=\left(\alpha_{1}, \ldots, \alpha_{\ell}\right) \in \mathbb{N}^{\ell}$ and $w \in \tilde{S}_{n}$ of length $|\alpha|:=\alpha_{1}+\cdots+\alpha_{\ell}$, an affine factorization of $w$ of weight $\alpha$ is a decomposition of the form $w=w^{\ell} \cdots w^{1}$, where $w^{i}$ is a cyclically decreasing permutation of length $\alpha_{i}$ for each $1 \leq i \leq \ell$. We denote the set of affine factorizations of $w$ by $\mathcal{W}_{w}$, and the subset of these having weight $\alpha$ is $\mathcal{W}_{w, \alpha}$. Their enumeration is $\mathcal{K}_{w, \alpha}=\left|\mathcal{W}_{w, \alpha}\right|$ for any $w \in \tilde{S}_{n}$.

Generating functions of affine factorizations were considered by Lam in [14] as affine Stanley symmetric functions. These are defined for any $\tilde{w} \in \tilde{S}_{n}$ by $F_{\tilde{w}}(x)=F_{\tilde{w}}=\sum_{w^{\ell \cdots w^{1}} \in \mathcal{W}_{\tilde{w}}} x_{1}^{\ell\left(w^{1}\right)} \cdots x_{\ell}^{\ell\left(w^{\ell}\right)}$. When $w \in S_{n}$ these are precisely the Stanley symmetric functions [32].

Define the content of a permutation $w \in \tilde{S}_{n}$ as $\operatorname{con}(w)=\left\{i \in[n] \mid s_{i}\right.$ appears in a reduced word for $\left.w\right\}$ This set can be obtained from a single reduced word for $w$ and is independent of the reduced word chosen. Moreover, a cyclically decreasing permutation is uniquely determined by its content. Hence, we often abuse notation and write the cyclically decreasing words for the actual permutation. 
Fix $x \in[n]$ and recall that $S_{\hat{x}}=\left\langle s_{0}, s_{1}, \ldots, \hat{s}_{x}, \ldots, s_{n-1}\right\rangle \subseteq \tilde{S}_{n}$. The characterization of our operators relies on the observation that when $u$ is a cyclically decreasing permutation, $\operatorname{con}(u)$ is strictly contained in $[n]$ and thus always lies in $S_{\hat{x}}$ for some $x \in[n]$. Therefore, for such a fixed $x$, there is a unique reduced word for $u$ given by the decreasing arrangement of entries in $\operatorname{con}(u)$ taken with respect to the order

$$
x-1>x-2>\cdots>0>n-1>\cdots>x+1 .
$$

\subsection{The crystal}

The operators $\tilde{e}_{r}, \tilde{f}_{r}$, and $\tilde{s}_{r}$ act by changing the $r$-th and $(r+1)$-st factors in a factorization for $w \in S_{\hat{x}}$ by altering the contents of these factors. The alteration is determined by a process of pairing reflections in the respective contents. Since the pairing is independent of $r$, we now define it for two factors.

For $x \in[n]$, consider cyclically decreasing permutations $u, v \in S_{\hat{x}}$. The uv-pairing with respect to $x$ is defined by pairing the largest $b \in \operatorname{con}(u)$ with the smallest $a>b$ in $\operatorname{con}(v)$ using the ordering as in (2). If there is no such $a$ in $v$ then $b$ is unpaired. The pairing proceeds in decreasing order on elements of $\operatorname{con}(u)$, and with each iteration previously paired letters of $\operatorname{con}(v)$ are ignored.

Example 3.1 For $n=14$, the $u v=\left(s_{9} s_{8} s_{5} s_{2} s_{12}\right)\left(s_{7} s_{6} s_{4} s_{1} s_{0} s_{13} s_{11}\right)$ pairing with respect to $x=3$ proceeds from left to right in the decreasing reordering of the entries of $\operatorname{con}(u) \operatorname{con}(v)$ with respect to $x$ :

$$
\left(2,12_{1}, 9_{2}, 8_{3}, 5_{4}\right)\left(1,0_{3}, 13_{1}, 11_{2}, 7,6_{4}, 4\right) .
$$

The crystal operators act by changing unpaired entries in adjacent factors of a factorization $w^{\ell} \cdots w^{1}$ of $w$. We thus define

$$
\begin{aligned}
& L_{r}\left(w^{\ell} \cdots w^{1}\right)=\left\{b \in \operatorname{con}\left(w^{r+1}\right) \mid b \text { is unpaired in the } w^{r+1} w^{r} \text {-pairing }\right\} \quad \text { and } \\
& R_{r}\left(w^{\ell} \cdots w^{1}\right)=\left\{b \in \operatorname{con}\left(w^{r}\right) \mid b \text { is unpaired in the } w^{r+1} w^{r} \text {-pairing }\right\},
\end{aligned}
$$

where the pairing is always taken with respect to a fixed $x \in[n]$.

Definition 3.2 Fix $x \in[n]$ and let $u, v \in S_{\hat{x}}$ be cyclically decreasing words. Then:

I) $\tilde{e}_{1}(u v)=\tilde{u} \tilde{v}$ is defined by the cyclically decreasing elements $\tilde{u}$ and $\tilde{v}$ where $\operatorname{con}(\tilde{u})=\operatorname{con}(u) \backslash\{b\}$ and $\operatorname{con}(\tilde{v})=\operatorname{con}(v) \cup\{b-t\}$ for $b=\min \left(L_{1}(u v)\right)$ and $t=\min \{i \geq 0 \mid b-i-1 \notin \operatorname{con}(u)\}$. If $L_{1}(u v)=\emptyset, \tilde{e}_{1}(u v)=0$.

II) $\tilde{f}_{1}(u v)=\tilde{u} \tilde{v}$ is defined by the cyclically decreasing elements $\tilde{u}$ and $\tilde{v}$ where $\operatorname{con}(\tilde{u})=\operatorname{con}(u) \cup\{a+s\}$ and $\operatorname{con}(\tilde{v})=\operatorname{con}(v) \backslash\{a\}$ for $a=\max \left(R_{1}(u v)\right)$ and $s=\min \{i \geq 0 \mid a+i+1 \notin \operatorname{con}(v)\}$. If $R_{1}(u v)=\emptyset, \tilde{f}_{1}(u v)=0$.

III) $\tilde{s}_{1}=\tilde{f}_{1}^{q-p}$ if $q>p$ and $\tilde{s}_{1}=\tilde{e}_{1}^{p-q}$ if $p>q$ where $p=\left|L_{1}(u v)\right|$ and $q=\left|R_{1}(u v)\right|$. When $p=q$, $\tilde{s}_{1}$ is the identity map.

The operators $\tilde{e}_{r}, \tilde{f}_{r}, \tilde{s}_{r}$ are defined to act on an affine factorization $w^{\alpha}:=w^{\ell(\alpha)} \cdots w^{1}$ for $w \in S_{\hat{x}}$ of weight $\alpha$ by $\tilde{e}_{r}\left(w^{\alpha}\right)=w^{\ell(\alpha)} \cdots \tilde{e}_{1}\left(w^{r+1} w^{r}\right) \cdots w^{1}, \tilde{f}_{r}\left(w^{\alpha}\right)=w^{\ell(\alpha)} \cdots \tilde{f}_{1}\left(w^{r+1} w^{r}\right) \cdots w^{1}$, and $\tilde{s}_{r}\left(w^{\alpha}\right)=w^{\ell(\alpha)} \cdots \tilde{s}_{1}\left(w^{r+1} w^{r}\right) \cdots w^{1}$.

Remark 3.3 Definition 3.2 is well-defined since the cyclically decreasing permutations $\tilde{u}$ and $\tilde{v}$ are uniquely defined by a strict subset of $[n]$ giving their contents. In particular, $\operatorname{con}(v) \cup\{b-t\} \subseteq[n] \backslash\{x\}$ since $x \notin \operatorname{con}(u) \cup \operatorname{con}(v)$ and $b-t \in \operatorname{con}(u)$ and similarly, con $(u) \cup\{a+s\} \subseteq[n] \backslash\{x\}$. 
Example 3.4 By abuse of notation we write cyclically decreasing words for affine permutations. For $n=$ 14 , consider the pairing with respect to $x=10$ of $u v=\left(9,8,5_{1}, 2_{2}, 12_{3}\right)\left(7,6_{1}, 4_{2}, 1,0,13_{3}, 11\right)$, so that $\tilde{e}_{1}(u v)=\left(9,5_{1}, 2_{2}, 12_{3}\right)\left(8,7,6_{1}, 4_{2}, 1,0,13_{3}, 11\right), \tilde{f}_{1}(u v)=\left(9,8,7,5_{1}, 2_{2}, 12_{3}\right)\left(6_{1}, 4_{2}, 1,0,13_{3}, 11\right)$, and $\tilde{s}_{1}(u v)=\left(9,8,7,5_{1}, 2_{2}, 1,12_{3}\right)\left(6_{1}, 4_{2}, 0,13_{3}, 11\right)$.

For $x \in[n]$ and any $w \in S_{\hat{x}}$, consider the graph $B(w)$ whose vertices are the affine factorizations $\mathcal{W}_{w}$ with at most $\ell$ factors and whose $I$-colored edges $x \stackrel{i}{\rightarrow} y$ for $x, y \in B(w)$ are determined by $\tilde{f}_{i} x=y$.

Theorem 3.5 For $x \in[n]$ and any $w \in S_{\hat{x}}, B(w)$ is a $U_{q}\left(A_{\ell-1}\right)$-crystal.

Consequently, by Theorem 2.2, the connected components of $B(w)$ are in bijection with highest weight vectors that are precisely defined below.

Definition 3.6 Fix $x \in[n], w \in S_{\hat{x}}$, and a composition $\alpha=\left(\alpha_{1}, \ldots, \alpha_{\ell}\right)$ with $|\alpha|=\ell(w)$. The factorization $w^{\alpha} \in \mathcal{W}_{w, \alpha}$ is highest weight when $\tilde{e}_{r} w^{\alpha}=\mathbf{0}$ for all $1 \leq r<\ell$. That is, $w^{\alpha}$ is highest weight if there is no unpaired residue in the pairing of $w^{r+1}$ and $w^{r}$ with respect to $x$ for all $1 \leq r \leq \ell-1$.

Example 3.7 The crystal $B\left(s_{3} s_{4} s_{1} s_{2}\right)$ of type $A_{2}$ has two highest weight elements $(1)\left(s_{3} s_{1}\right)\left(s_{4} s_{2}\right)$ and $\left(s_{1}\right)\left(s_{3}\right)\left(s_{4} s_{2}\right)$ of weights $(2,2)$ and $(2,1,1)$, respectively. This agrees with the Schur expansion of the Stanley symmetric function $F_{s_{3} s_{4} s_{1} s_{2}}=s_{(2,2)}+s_{(2,2,1)}$.

\section{Specht modules}

The crystal $B(w)$ for $w \in S_{n}$ corresponds to representations carrying an action of the symmetric group called Specht modules. These modules $\mathcal{S}^{D}$ are associated to finite subsets $D$ of $\mathbb{N} \times \mathbb{N}$ called diagrams. Their origin was in Young's work to explicitly produce the irreducible representations of the symmetric group. He required only Ferrers diagrams, the graphical depiction of a partition $\lambda=\left(\lambda_{1}, \ldots, \lambda_{m}\right)$ with non-increasing positive integer entries obtained by stacking rows of $\lambda_{i}$ boxes in the left corner (with its smallest row at the top). Here $\ell(\lambda):=m$ is called the length of the partition $\lambda$. The set of Specht modules indexed by Ferrers diagrams $\lambda$, where $|\lambda|=\sum_{i=1}^{m} \lambda_{i}=\ell$, is a complete set of irreducible $S_{\ell}$-modules.

It has since been established that other subclasses of Specht modules are fundamental as well. For example, Specht modules indexed by skew-shaped diagrams give $\mathfrak{s l}_{\ell-1}$-representations, and their decomposition as a direct sum of irreducible submodules

$$
\mathcal{S}^{\nu / \lambda}=\bigoplus_{\mu} c_{\lambda, \mu}^{\nu} \mathcal{S}^{\mu}
$$

yields multiplicities $c_{\lambda, \mu}^{\nu}$ that are given by the acclaimed Littlewood-Richardson (LR) rule. Another notable family consists of the Specht modules indexed by Rothe diagrams of permutations, defined uniquely for each $w \in S_{n}$ to be $D(w)=\{(i, w(j)) \mid 1 \leq i<j \leq n, w(i)>w(j)\}$.

\subsection{Specht modules and crystals for skew shapes}

A foundational example in crystal theory is the $\mathfrak{s l}_{\ell-1}$-crystal [21, 22, 11] on skew tableaux which, by Schur-Weyl duality, can be associated to the Specht modules $\mathcal{S}^{D}$ for skew shapes $D$. Our point of departure is to recall the crystal on tableaux and to show that it is a special case of $B(w)$ on affine factorizations.

The vertices of the $\mathfrak{s l}_{\ell-1}$-crystal crystal $B(\nu / \lambda)$ consist of the semi-standard skew tableaux $\operatorname{SSYT}(\nu / \lambda)$ over the alphabet $\{1,2, \ldots, \ell\}$. Here $t \in \operatorname{SSYT}(\nu / \lambda)$ when it is a filling of the diagram $D=\nu / \lambda$ with 
letters placed non-decreasing across rows and increasing up columns. Its weight is defined by the composition $\mu=\left(\mu_{1}, \ldots, \mu_{\ell}\right)$ where $\mu_{i}$ records the number of times $i$ occurs in $t$.

Crystal operators $\tilde{e}_{i}$ and $\tilde{f}_{i}$ for $1 \leq i<\ell$ are defined on $t \in \operatorname{SSYT}(\nu / \lambda)$ using a bracketing of the letters $i$ and $i+1$ in $t$. Scan the columns of $t$ from right to left, bottom to top. When a letter $i+1$ appears, pair it with the closest previously scanned $i$ in this scanning order that has not yet been paired (if possible). Then $\tilde{f}_{i}(t)$ is the skew tableau obtained from $t$ by changing the rightmost unpaired $i$ into an $i+1$. If none exists, $\tilde{f}_{i}(t)=\mathbf{0}$. Similarly, $\tilde{e}_{i}(t)$ is obtained from $t$ by changing the leftmost unpaired $i+1$ into an $i$ and if none exists, $\tilde{e}_{i}(t)=\mathbf{0}$.

Recall that Theorem 2.2 indicates that highest weights correspond to irreducible components. In this setting, $t \in B(\nu / \lambda)$ is highest weight if $\tilde{e}_{i} t=\mathbf{0}$ for all $1 \leq i<\ell$ and thus the multiplicities in (3) are given by the following combinatorial objects.

Crystal version of Littlewood-Richardson Rule.

$c_{\lambda, \mu}^{\nu}$ is the number of semi-standard tableaux $t$ of shape $\nu / \lambda$ and weight $\mu$ such that $t$ is highest weight.

The crystal $B(w)$ introduced in Section 3 reduces to the crystal $B(\nu / \lambda)$ when $w \in S_{n} \subset \tilde{S}_{n}$ is 321 avoiding. Recall that $w \in S_{n}$ is 321 -avoiding if none of its reduced words contain a braid $s_{i} s_{i+1} s_{i}$. This subclass of permutations is in bijection [1] with skew shapes fitting inside a rectangle by removing all rows and columns without cells from the Rothe diagram $D(w)$.

Proposition 4.1 If $w \in S_{n}$ is 321-avoiding, let $D(w)=\nu / \lambda$ be the corresponding skew shape. As $U_{q}\left(\mathfrak{s l}_{\ell-1}\right)$-crystals, $B(\nu / \lambda)$ over the alphabet $\{1,2, \ldots, \ell\}$ is isomorphic to $B(w)$ with at most $\ell$ factors.

\subsection{Specht modules and crystals for Rothe diagrams}

For any affine permutation $\tilde{w} \in S_{\hat{x}}$, the crystal $B(\tilde{w})$ on affine factorizations of $\tilde{w}$ gives the structure of more general Specht modules. Since $S_{\hat{x}}$ for any $x \in[n]$ is isomorphic to $S_{n}$, we can associate a permutation $\tau_{x}(\tilde{w})$ in $S_{n}$ to each $\tilde{w} \in S_{\hat{x}}$ by shifting the generators of $\tilde{w}$ by $-x \bmod n$. We thus define the diagram of $\tilde{w}$ to be $\tilde{D}(\tilde{w}):=D\left(\tau_{x}(\tilde{w})\right)$. The crystal $B(\tilde{w})$ on affine factorizations of $\tilde{w} \in S_{\hat{x}}$ gives the structure of the modules $\mathcal{S}^{\tilde{D}(\tilde{w})}$.

Theorem 4.2 For any $\tilde{w} \in S_{\hat{x}} \subset \tilde{S}_{n}$ with $x \in[n]$, the decomposition of the Specht module $\mathcal{S}^{\tilde{D}(\tilde{w})}$ into irreducible submodules is $\mathcal{S}^{\tilde{D}(\tilde{w})}=\bigoplus_{\lambda} a_{\tilde{w}, \lambda} \mathcal{S}^{\lambda}$, where the multiplicity $a_{\tilde{w}, \lambda}$ is the number of highest weight factorizations in $\mathcal{W}_{\tilde{w}, \lambda}$.

This result can also be interpreted on the level of symmetric functions by noting that the Schur functions $s_{\lambda}$ are characters of the irreducible Specht modules $\mathcal{S}_{\lambda}$.

Corollary 4.3 For any $\tilde{w} \in S_{\hat{x}} \subset \tilde{S}_{n}$ with $x \in[n]$, or for any $\tilde{w} \in \tilde{S}_{n}$ and $\ell(\lambda) \leq 2$, the coefficient $a_{\tilde{w}, \lambda}$ in $F_{\tilde{w}}=\sum_{\lambda} a_{\tilde{w}, \lambda} s_{\lambda}$ enumerates the highest weight factorizations in $\mathcal{W}_{\tilde{w}, \lambda}$.

Edelman and Greene [4] (see also [6, Theorem 1.2]) characterized the coefficients $a_{w, \lambda}$ of a Schur function $s_{\lambda}$ in the Stanley symmetric function $F_{w}(x)$ for $w \in S_{n}$. For any $w \in S_{n}$, they proved that $a_{w, \lambda}$ is the number of semi-standard tableaux of shape $\lambda^{\prime}$ (the transpose of $\lambda$ ) whose column-reading word is a reduced word of $w$. Theorem 4.2 thus gives a different formulation for these coefficients.

Corollary 4.4 For any permutation $\tilde{w} \in S_{\hat{x}} \subset \tilde{S}_{n}$ and partition $\lambda$, there is a bijection between the highest weight factorizations $\left\{v^{\ell} \cdots v^{1} \in \mathcal{W}_{\tilde{w}, \lambda} \mid \tilde{e}_{i}\left(v^{\ell} \cdots v^{1}\right)=\mathbf{0}\right.$ for all $\left.1 \leq i<\ell\right\}$, and the semi-standard tableaux of shape $\lambda^{\prime}$ whose column-reading word is a reduced word of $\tilde{w}$. 
The bijection mentioned in Corollary 4.4 is given explicitly by a variant of the Edelman-Greene (EG) insertion [4]. In fact, it can be extended to the full crystal (not just highest weight elements) as follows. Given an affine factorization $v^{\ell} \cdots v^{1} \in \mathcal{W}_{\tilde{w}}$, consider $\bar{v}^{1} \cdots \bar{v}^{\ell}$ by reversing all factors. In particular, each cyclically decreasing factor $v^{i}$ turns into a cyclically increasing factor $\bar{v}^{i}$. Now successively insert the factors $\bar{v}^{i}$ for $i=1,2, \ldots, \ell$ using the EG insertion $\varphi_{\mathrm{EG}}: v^{\ell} \cdots v^{1} \mapsto(P, Q)$, where $P$ is the EG insertion tableau and $Q$ is the EG recording tableau. Then, according to the next theorem, the bijection of Corollary 4.4 is explicitly the transpose of the insertion tableau $\varphi_{\mathrm{EG}}^{P}\left(v^{\ell} \cdots v^{1}\right)=P$ of the highest weight element $v^{\ell} \cdots v^{1}$.

Theorem 4.5 For any permutation $\tilde{w} \in S_{\hat{x}} \subset \tilde{S}_{n}$, the crystal isomorphism $B(\tilde{w}) \cong \bigcup_{\lambda} B(\lambda)$, where each $B(\lambda)$ occurs with multiplicity $a_{\tilde{w} \lambda}$, is explicitly given by $\varphi_{\mathrm{EG}}^{Q}\left(v^{\ell} \cdots v^{1}\right)=Q$.

\section{Highest weights and geometric invariants}

Representatives for homology classes of the affine Grassmannian of $S L_{n}$ are given by a basis for the subring $\Lambda_{(n)}=\mathbb{Z}\left[h_{1}, \ldots, h_{n-1}\right]$ of the ring of symmetric functions $\Lambda$, where $h_{r}=\sum_{i_{1} \leq \cdots \leq i_{r}} x_{i_{1}} \cdots x_{i_{r}}$ is the $r$-th homogeneous symmetric function. The basis is comprised of symmetric functions $s_{u}^{(k)}$ called $k$-Schur functions indexed by $u \in \tilde{S}_{n}^{0}$ (where hereafter, $k=n-1$ ).

The importance of this basis to our study is that the Schubert structure constants for $H_{*}(\mathrm{Gr})$ match the coefficients in (1), and intensely studied families of constants arise as subsets of these affine LittlewoodRichardson coefficients. By identifying affine Stanley symmetric functions with a family of dual $k$-Schur functions, we can apply the crystal on affine factorizations of Section 3 to the study of affine LR numbers.

\subsection{The affine Stanley/dual $k$-Schur correspondence}

There are many equivalent formulations for the $k$-Schur basis. In the spirit of our presentation, we use its characterization by the expansion in terms of the homogeneous basis $\left\{h_{\lambda}\right\}_{\lambda \in \mathcal{P}^{n}}$, where $h_{\lambda}=h_{\lambda_{1}} \cdots h_{\lambda_{\ell}}$ and $\mathcal{P}^{n}=\left\{\lambda \mid \lambda_{1}<n\right\}$ is the set of partitions with all parts shorter than $n$.

For each $w \in \tilde{S}_{n}^{0}$ that is affine Grassmannian, the matrix $\mathcal{K}$ whose entries $\mathcal{K}_{w, \mu}=\left|\mathcal{W}_{w, \mu}\right|$ enumerate affine factorizations of $w$ with specified weight is square and unitriangular. We let $\overline{\mathcal{K}}=\mathcal{K}^{-1}$ and define the $k$-Schur function to be $s_{w}^{(k)}=\sum_{\mu \in \mathcal{P}^{n}} \overline{\mathcal{K}}_{\mu, w} h_{\mu}$. To clarify that the matrix is square, recall that the set of affine Grassmannian elements $\tilde{S}_{n}^{0}$ is in bijection with $\mathcal{P}^{n}$. We use a correspondence [2] coming from the inversions of an affine element. For any affine element $w$, its left inversion vector (the affine Lehmer code) $\operatorname{linv}(w)=\left(\alpha_{1}, \ldots, \alpha_{n}\right)$ is a composition of length $n$ where $\alpha_{i}$ is the number of positions $-\infty<j<i$ such that $w(j)>w(i)$. Since the window of an affine Grassmannian $w \in \tilde{S}_{n}^{0}$ is increasing, $\operatorname{linv}(w)$ is weakly decreasing and its last entry is zero. In fact, the map $\mathcal{L C}: \tilde{S}_{n}^{0} \rightarrow \mathcal{P}^{n}$, where

$$
\mathcal{L C}: w \mapsto \operatorname{linv}(w)^{\prime}
$$

is a bijection. We use $w_{\lambda}$ to denote the affine permutation whose image under $\mathcal{L C}$ is $\lambda$.

Example 5.1 For $w=[-2,0,1,4,12] \in \tilde{S}_{5}^{0}$, the left inversion vector is $\operatorname{linv}(w)=(3,2,2,1,0)$ and its conjugate is $\mathcal{L C}(w)=(4,3,1) \in \mathcal{P}^{5}$. Thus, in our notation, $w=w_{(4,3,1)}$.

Although the algebra of $\Lambda_{(n)}$ is not a self dual Hopf algebra, it is dual to the quotient $\Lambda^{(n)}=\Lambda /\left\langle m_{\lambda}\right|$ $\left.\lambda_{1} \geq n\right\rangle$, where $m_{\lambda}=\sum x_{\alpha_{1}}^{\lambda_{1}} x_{\alpha_{2}}^{\lambda_{2}} \cdots x_{\alpha_{\ell}}^{\lambda_{\ell}}$ over tuples $\left(\alpha_{1}, \ldots, \alpha_{\ell}\right) \in \mathbb{N}^{\ell}$ with distinct entries. The 
elements $m_{\lambda}$ with $\lambda \in \mathcal{P}^{n}$ may be chosen as representatives of the dual algebra. We use duality to produce a second basis, now for the algebra $\Lambda^{(n)}$. We appeal to a pairing $\langle\cdot, \cdot\rangle: \Lambda_{(n)} \times \Lambda^{(n)} \rightarrow \mathbb{Q}$, where $h_{\mu} \in \Lambda_{(n)}$ and $m_{\lambda} \in \Lambda^{(n)}$ are dual elements, that is, $\left\langle h_{\mu}, m_{\lambda}\right\rangle=\delta_{\lambda \mu}$.

Under this pairing, the dual $k$-Schur functions are the elements $\left\{\mathcal{F}_{\lambda}\right\}_{\lambda \in \mathcal{P}^{n}}$ forming the unique basis of the subspace of $\Lambda^{(n)}$ dual to the $k$-Schur basis. In fact, dual $k$-Schur functions were introduced in greater generality for any skew diagram in $\mathcal{D}=\left\{\nu / \lambda \mid \ell\left(w_{\nu} w_{\lambda}^{-1}\right)=\ell\left(w_{\nu}\right)-\ell\left(w_{\lambda}\right)\right\}$ to be $\mathcal{F}_{\nu / \lambda}=$ $\sum_{\mu} \mathcal{K}_{w_{\nu} w_{\lambda}^{-1}, \mu} m_{\mu}$, and it was shown in [19] that these can instead be used to produce the affine LRcoefficients by the expansion

$$
\mathcal{F}_{\nu / \lambda}=\sum_{\mu} c_{w_{\lambda}, w_{\mu}}^{w_{\nu}, k} \mathcal{F}_{\mu}
$$

Lam [14] introduced affine Stanley symmetric functions $F_{\tilde{w}}$ for $\tilde{w} \in \tilde{S}_{n}$ and proved that $\mathcal{F}_{\nu / \lambda}=F_{w_{\nu} w_{\lambda}^{-1}}$.

The converse was not readily apparent at the time, but it turns out that every affine Stanley symmetric function is a skew dual $k$-Schur function. We discovered a correspondence between arbitrary affine elements and skew diagrams. It is most naturally formulated using $\tilde{S}_{n,\left(\begin{array}{l}n \\ 2\end{array}\right)}$, the set of bijections from $\mathbb{Z}$ to $\mathbb{Z}$ with $w(i+n)=w(i)+n$ for all $i \in \mathbb{Z}$ and $\sum_{i=1}^{n}(w(i)-i)=\left(\begin{array}{c}n \\ 2\end{array}\right) n$. In [20], a map from permutations of $S_{n}$ to affine elements of $\tilde{S}_{n,\left(\begin{array}{c}n \\ 2\end{array}\right)}$ defined by $\mathfrak{a} \mathfrak{f}: w \mapsto[w(1), w(2)+n, w(3)+2 n, \ldots, w(n)+(n-1) n]$ is the crux of the association of Gromov-Witten invariants for flag manifolds with affine LR-coefficients (details are given in Section 5.3. This same map ties affine elements to skew diagrams.

Definition 5.2 For $\tilde{w} \in \tilde{S}_{n}$, consider the affine Grassmannian element $\tilde{v}$ determined by rearranging the window of $\tilde{w}$ and $u \in S_{n}$ such that $\tilde{w}=\tilde{v} u$ and $\ell(\tilde{w})=\ell(\tilde{v})+\ell(u)$. Let $\kappa: \tilde{w} \mapsto \nu / \lambda$, where $\nu=\mathcal{L C}(\tilde{v} \mathfrak{a f}(\mathrm{id}))$ and $\lambda=\mathcal{L C}\left(\mathfrak{a f}\left(u^{-1}\right)\right)$ with $\mathcal{L C}$ of (4) naturally extended to affine Grassmannian elements in $\widetilde{S}_{n,\left(\begin{array}{l}n \\ 2\end{array}\right)}$.

Proposition 5.3 Let $\tilde{w} \in \tilde{S}_{n}$ and $\kappa(\tilde{w})=\nu / \lambda$. Then $\tilde{w}$ equals $w_{\nu} w_{\lambda}^{-1}$ up to a cyclic relabeling of the generators and $\ell(\tilde{w})=\ell\left(w_{\nu}\right)-\ell\left(w_{\lambda}\right)$.

Corollary 5.4 For any $\tilde{w} \in \tilde{S}_{n}, F_{\tilde{w}}=\mathcal{F}_{\kappa(\tilde{w})}$.

\subsection{Structure constants for $H_{*}(\mathrm{Gr})$}

We are now in a position to describe Schubert structure constants in the homology of Gr as highest weights of the crystal $B(\tilde{w})$. The crystal applies to a subclass of constants in the $k$-Schur expansion of products of a $k$-Schur function with a Schur function indexed by any $\mu \subset\left(r^{n-r}\right)$, for some $1 \leq r<n$. A fundamental property of $k$-Schur functions tells us these indeed are homology structure constants. That is, when $\mu \subset\left(r^{n-r}\right)$ for some $1 \leq r<n, s_{w_{\mu}}^{(k)}=s_{\mu}$. The coefficients are thus a subclass of the affine LR-coefficients in (1).

We first revisit the affine Stanley symmetric functions with this restriction in hand. While (5) explains that the dual $k$-Schur expansion of $F_{\tilde{w}}$ yields positive coefficients, it does not suggest the same about the expansion in terms of Schur functions $s_{\mu}$. In fact, the Schur coefficients are not positive in general. However, these are Gromov-Witten invariants for flags when $\mu \subset\left(r^{n-r}\right)$.

Proposition 5.5 Let $\tilde{w} \in \tilde{S}_{n}$. For each $\mu \subset\left(r^{n-r}\right)$ for some $1 \leq r<n$, the coefficient $a_{\tilde{w}, \mu}$ in $F_{\tilde{w}}=\sum_{\mu} a_{\tilde{w}, \mu} s_{\mu}$ is the affine LR-coefficient $c_{w_{\mu}, w_{\lambda}}^{w_{\nu}, k}$, where $\kappa(\tilde{w})=\nu / \lambda$. 
To use the crystal for the greatest generality of results, we appeal to another fundamental property of $k$-Schur functions. We are concerned with distinguished affine elements $R_{i}$ that can be defined by their action on $u \in \tilde{S}_{n}$ in window notation, $R_{i} u=\left[u_{1}-(n-i), u_{2}-(n-i), \ldots, u_{i}-(n-i), u_{i+1}+\right.$ $\left.i, \ldots, u_{n}+i\right]$, for $1 \leq i<n$. Because $R_{i}(\mathrm{id})=w_{\left(i^{n-i}\right)}$, the $R_{i}:=R_{i}(\mathrm{id})$ are called $k$-rectangles. In this case, the $k$-Schur function $s_{R_{i}}^{(k)}$ is simply $s_{\left(i^{n-i}\right)}$. We shall extensively use that the multiplication of a $k$-Schur function by such a term is trivial. By [18, Theorem 40], $s_{R_{i}}^{(k)} s_{u}^{(k)}=s_{R_{i} u}^{(k)}$.

Theorem 5.6 Consider $v, w \in \tilde{S}_{n}^{0}$ and $\mu \subset\left(r^{n-r}\right)$ for any $1 \leq r<n$. If $\ell(v)-\ell(w) \neq|\mu|$ then $c_{R w_{\mu}, w}^{R v, k}=0$. Otherwise, if either $(i) v w^{-1} \in S_{\hat{x}}$ for some $x \in[n]$ or $(i i) \ell(\mu)=2$, then for any product of $k$-rectangles $R=\prod R_{i}$

$$
c_{R w_{\mu}, w}^{R v, k}=\# \text { of highest weight factorizations of } v^{-1} \text { of weight } \mu .
$$

\subsection{Flag Gromov-Witten invariants}

Let $\mathrm{Fl}_{n}$ be the complete flag manifold (chains of vector spaces) in $\mathbb{C}^{n} . \mathrm{Fl}_{n}$ decomposes into Schubert cells which are indexed by permutations $w \in S_{n}$. As a linear space, the quantum cohomology of $\mathrm{Fl}_{n}$ is $\mathrm{QH}^{*}\left(\mathrm{Fl}_{n}\right)=H^{*}\left(\mathrm{Fl}_{n}\right) \otimes \mathbb{Z}\left[q_{1}, \ldots, q_{n-1}\right]$ for parameters $q_{1}, \ldots, q_{n-1}$. The multiplicative structure, specializing to $H^{*}\left(\mathrm{Fl}_{n}\right)$ when $q_{1}=\cdots=q_{n-1}=0$, is defined by $\sigma_{u} * \sigma_{w}=\sum_{v} \sum_{d} q^{d}\langle u, w, v\rangle_{d} \sigma_{w_{0} v}$ where $q^{d}=q_{1}^{d_{1}} \cdots q_{n-1}^{d_{n-1}}, w_{0}$ is the longest element in $S_{n}$, and the structure constants $\langle u, w, v\rangle_{d}$ are the 3-point Gromov-Witten invariants of genus 0 which count equivalence classes of certain rational curves in $\mathrm{Fl}_{n}$. The combinatorial study of these Gromov-Witten invariants for flag manifolds is ongoing. In the simplest case when $q_{1}=\cdots=q_{n-1}=0$, a manifestly positive formula has been actively sought after for the last 40 years.

Our approach is to use the identification of these Gromov-Witten invariants with affine LR-coefficients from [20]. As this requires the map af : $S_{n} \rightarrow \tilde{S}_{n,\left(\begin{array}{l}n \\ 2\end{array}\right)}$, we shall allow for $k$-Schur functions indexed by affine Grassmannian elements of $\tilde{S}_{n,\left(\begin{array}{c}n \\ 2\end{array}\right)}$ via the isomorphism $\tilde{S}_{n,\left(\begin{array}{c}n \\ 2\end{array}\right)} \cong \tilde{S}_{n}$. It was shown in [20] that degree $d$, 3-point Gromov-Witten invariants for complete flags are the affine LR-coefficients given by $\langle u, w, v\rangle_{d}=c_{\mathfrak{a f}(u), \mathfrak{a} \mathfrak{f}(w)}^{\mathfrak{a f}_{d}(v), k}$, where $\mathfrak{a f}_{d}(v)=\left[a_{1}, \ldots, a_{n}\right]$ is defined by setting $a_{i}=v(i)+(i-1) n+\left(\begin{array}{l}i \\ 2\end{array}\right)-$ $\left(\begin{array}{c}n+1-i \\ 2\end{array}\right)+n d_{i}-n d_{i-1}$.

We can thus apply Theorem 5.6 to the study of Gromov-Witten invariants. It turns out that the imposed conditions translate to the study of the natural subclass of $\langle u, w, v\rangle_{d}$ where $u$ is a representative of $S_{n} / S_{r} \times S_{n-r}$ for some $r$. Recall that these permutations are called Grassmannian, characterized as the permutations of $S_{n}$ with exactly one descent in position $r$. Such a permutation $u$ can be identified with a partition $\lambda(u)=\left(\lambda_{1}, \ldots, \lambda_{r}\right)$ in the $r \times(n-r)$ rectangle by setting $\lambda_{i}=\mid\left\{u_{j} \mid u_{r+1-i}>u_{j}\right.$ and $\left.j>r\right\} \mid$. Denote the complement partition to $\lambda$ in the rectangle by $\lambda^{\vee}=\left(n-r-\lambda_{r}, \ldots, n-r-\lambda_{1}\right)$; that is, $\lambda^{\vee}$ is obtained from $\lambda$ by taking the complement of $\lambda$ in the $r \times(n-r)$-rectangle and rotating it by $180^{\circ}$.

Theorem 5.7 Let $d \in \mathbb{N}^{n-1}$ and $u, w, v \in S_{n}$ where $u$ is Grassmannian with descent at position $r$. Define $\mu=\left(\lambda(u)^{\vee}\right)^{\prime}$ and $\tilde{v}=\left[\tilde{v}_{1}, \ldots, \tilde{v}_{n}\right]$ with $\tilde{v}_{i}=v(i)+(i-2) n+r+n d_{i}-n d_{i-1}$ for $i \leq r$ and $\tilde{v}_{i}=v(i)+(i-1) n+r+n d_{i}-n d_{i-1}$ otherwise. If $(i) \tilde{v} \mathfrak{a f}(w)^{-1} \in S_{\hat{x}}$ for some $x \in[n]$ or $(i i) \ell(\mu)=2$, then

$$
\langle u, w, v\rangle_{d}=\# \text { of highest weight factorizations of } \tilde{v} \mathfrak{a f}(w)^{-1} \text { of weight } \mu,
$$

unless $\ell(\tilde{v}) \neq \ell\left(w_{\mu}\right)+\ell(\mathfrak{a} \mathfrak{f}(w))$, in which case, $\langle u, w, v\rangle_{d}=0$. 
The result directly applies to the problem of describing structure constants in the product of a Schur polynomial by a (quantum) Schubert polynomial [23, 5]. Recall that when $u \in S_{n}$ is Grassmannian with a descent at position $r$, the Schubert polynomial $\mathfrak{S}_{u}$ is simply the Schur polynomial $s_{\lambda(u)}\left(x_{1}, \ldots, x_{r}\right)$. Therefore, here we are addressing the coefficients in $s_{\lambda}\left(x_{1}, \ldots, x_{r}\right) \mathfrak{S}_{w}=\sum_{v \in S_{n}}\langle u, w, v\rangle_{0} \mathfrak{S}_{w_{0} v}$, and in the quantum analog $(d \neq 0)$. Note that the cases treated here are different from the ones treated in [26].

Corollary 5.8 Let $u, v, w \in S_{n}$ where $u$ has exactly one descent at position $r$ and set $\mu=\left(\lambda(u)^{\vee}\right)^{\prime}$. If $|\mu| \neq r(n-r)+\ell(w)-\ell(v)$, then $\langle u, w, v\rangle_{0}=0$. Otherwise if $\left(R_{r} v\right) w^{-1} \in S_{\hat{x}}$ for some $x \in[n]$ or $\ell(\mu)=2$, then $\langle u, w, v\rangle_{0}=\#$ of highest weight factorizations of $\left(R_{r} v\right) w^{-1}$ of weight $\mu$.

\subsection{Fusion coefficients and positroid stratification}

Our methods using the crystal $B(\tilde{w})$ for $w \in S_{\hat{x}}$ for some $x \in[n]$ also apply to the Verlinde (fusion) algebra of the WZW model associated to $\widehat{s u}(\ell)$ at level $n-\ell$ and the decomposition of a positroid variety into Schubert varieties of $\operatorname{Gr}(r, n)$. Details are given in the long version of this paper.

\section{References}

[1] S. Billey, W. Jockusch, R. Stanley, Some combinatorial properties of Schubert polynomials, J. of Alg. Comb. 2 (1993), 345-374.

[2] A. Björner, F. Brenti, Affine permutations of type A, Electron. J. Combin. 3 (1996), Research Paper 18, 35 pp.

[3] R. Bott, The space of loops on a Lie group, Michigan Math. J. 5 (1958), 35-61.

[4] P. Edelman, C. Greene, Balanced tableaux, Adv. in Math. 63 (1987), 42-99.

[5] S. Fomin, S. Gelfand, A. Postnikov, Quantum Schubert polynomials, J. Amer. Math. Soc. 10 (1997), 565-596.

[6] S. Fomin, C. Greene, Noncommutative Schur functions and their applications, Discr. Math. 193 (1998), $179-200$.

[7] H. Garland, M. .S. Raghunathan, A Bruhat decomposition for the loop space of a compact group: a new approach to results of Bott, Proc. Nat. Acad. Sci. U.S.A. 72 (1975), 4716-4717.

[8] M. Kashiwara, Crystalizing the q-analogue of universal enveloping algebras, Comm. Math. Phys. 133 (1990), 249-260.

[9] M. Kashiwara, On crystal bases of the q-analogue of universal enveloping algebras, Duke Math. J. 63 (1991), 465-516.

[10] M. Kashiwara, On crystal bases, Representations of groups (Banff, AB, 1994), 155-197, CMS Conf. Proc., 16, Amer. Math. Soc., Providence, RI, 1995.

[11] M. Kashiwara, T. Nakashima, Crystal graphs for representations of the q-analogue of classical Lie algebras, J. Algebra 165 (1994), 295-345.

[12] B. Kostant, S. Kumar, The nil Hecke ring and cohomology of G/P for a Kac-Moody group G, Adv. in Math. 62 (1986), 187-237.

[13] M. Kontsevich, The moduli space of curves, Progr. Math. 129 (1995) Birkhäuser Boston, Boston, MA, 335-368.

[14] T. Lam, Affine Stanley symmetric functions, Amer. J. Math. 128 (2006), 1553-1586. 
[15] T. Lam, Schubert polynomials for the affine Grassmannian, J. Amer. Math. Soc. 21 (2008), 259-281.

[16] T. Lam, M. Shimozono, Quantum cohomology of G/P and homology of affine Grassmannian, Acta Math. 204 (2010), 49-90.

[17] L. Lapointe, A. Lascoux, J. Morse, Tableau atoms and a new Macdonald positivity conjecture, Duke Math. J. 116 (2003), 103-146.

[18] L. Lapointe, J. Morse, A k-tableau characterization of k-Schur functions, Adv. Math. 213 (2007), $183-204$.

[19] L. Lapointe, J. Morse, Quantum cohomology and the k-Schur basis, Trans. AMS 360 (2008), 2021-2040.

[20] L. Lapointe, J. Morse, Flag Gromov-Witten invariants and k-Littlewood Richardson coefficients, in preparation.

[21] A. Lascoux, M.-P. Schützenberger, Sur une conjecture de H. O. Foulkes, C. R. Acad. Sci. Paris Sér. A-B 286 (1978), A323-A324.

[22] A. Lascoux, M.-P. Schützenberger, Croissance des polynômes de Foulkes-Green, C. R. Acad. Sci. Paris Sér. A-B 288 (1979), A95-A98.

[23] A. Lascoux, M.-P. Schützenberger, Polynômes de Schubert, C. R. Acad. Sci. Paris Sér. I Math. 294 (1982), 447-450.

[24] P. Littelmann, A Littlewood-Richardson rule for symmetrizable Kac-Moody algebras, Invent. Math. 116 (1994), 329-346.

[25] D.E. Littlewood, A. R Richardson, Group characters and algebra, Philos. Trans. R. Soc. Lond. Ser. A 233 (1934) 99-141.

[26] K. Mészáros, G. Panova, A. Postnikov, Schur times Schubert via the Fomin-Kirillov algebra, The Electronic Journal of Combinatorics 21 (1) (2014), P1.39.

[27] T. Nakashima, Crystal base and a generalization of the Littlewood-Richardson rule for the classical Lie algebras, Comm. Math. Phys. 154 (1993), 215-243.

[28] D. Peterson, Quantum cohomology of $G / P$, Lecture notes, M.I.T., Spring 1997.

[29] W. A. Stein et al. Sage Mathematics Software (Version 5.12). The Sage Development Team, 2013. http: //www.sagemath.org.

[30] The Sage-Combinat community. Sage-Combinat: enhancing Sage as a toolbox for computer exploration in algebraic combinatorics, 2008. http: / / combinat. sagemath. org.

[31] M.-P. Schützenberger, La correspondance de Robinson, Combinatoire et représentation du groupe symétrique (Actes Table Ronde CNRS, Univ. Louis-Pasteur Strasbourg, Strasbourg, 1976), pp. 59-113. Lecture Notes in Math., Vol. 579, Springer, Berlin, 1977.

[32] R.P. Stanley, On the number of reduced decompositions of elements of Coxeter groups, Euro. J. Combin. 5 (1984), 359-372.

[33] J. R. Stembridge, A local characterization of simply-laced crystals, Trans. AMS 355 (2003), 4807-4823.

[34] E. Witten, Quantum cohomology in string theory, Comm. Math. Phys. 118 (1988), 411-449. 